\title{
Overview and outlook on muon survey tomography based on micromegas detectors for unreachable sites technology
}

\author{
I. Lázaro Roche $e^{1,2,3, a}$, T. Serre ${ }^{1}$, J.B. Decitre ${ }^{2}$, A. Bitri ${ }^{3}$, C. Truffert ${ }^{1}$, and S. Gaffet ${ }^{2}$ \\ ${ }^{1}$ Iris Instruments, 1 avenue buffon, BP. 16007, 45060 Orléans Cedex 2, France \\ ${ }^{2}$ LSBB UMS3538, La grande combe, 84400 Rustrel, France \\ ${ }^{3}$ BRGM, 3 avenue Claude-Guillemin, BP. 36009, 45060 Orléans Cedex 2, France
}

\begin{abstract}
The present document describes the functioning principles of the Muon Survey Tomography based on Micromegas detectors for Unreachable Sites Technology and its distinguishing features from other Micromegas-like detectors. Additionally, it addresses the challenges found while operating the first generation and the resulting improvements. Currently, the project Temporal Tomography of the Densitometry by the Measurement of Muons is focused on obtaining a reliable pulse from the micromesh, associated to the passing of a muon, in order to trigger the acquisition and operate in standalone mode. An outlook of the future steps of the project is provided as well.
\end{abstract}

\section{Introduction}

Muons are charged particles originated naturally all around us. Primary cosmic rays from deep space hit the upper atmosphere and produce secondary particles, including muons. Muons are heavy, (207 times more massive than the $\mathrm{e}^{-}$), fast (99.97\% of the speed of light) and they lack of strong interaction. These characteristics allow them to propagate up to several hundreds of meters underground, with an absorption proportional to the amount of matter they travel trough. Muon tomography, or muography, leans on the drop of the muon population as depth goes on to calculate the density of the matter crossed [1,2].

The Temporal Tomography of the Densitometry by the Measurement of Muons collaboration (T2DM2) has developed a new tool to measure the muon's flux and trajectories in order to image dynamically the densitometry of the medium. The MUST ${ }^{2}$ camera is born from the inclusion of a Micromegas detector [3, 4] within a Time Projection Chamber. This combination presents interesting distinctive features compared with the regular Micromegas detectors, such as the capability to reconstruct the particle's trajectory with only one detection plane. This reduces substantially the volume and weight of the camera, specially compared to other muon detection systems, such as scintillators.

The technology validation for this new tool is being carried out by the Low Noise Inter Disciplinary Underground Science and Technology URL (LSBB) with the prototypes developed between 2012 and 2015.

\footnotetext{
${ }^{a}$ Corresponding author: ignacio.lazaro@lsbb.eu
} 


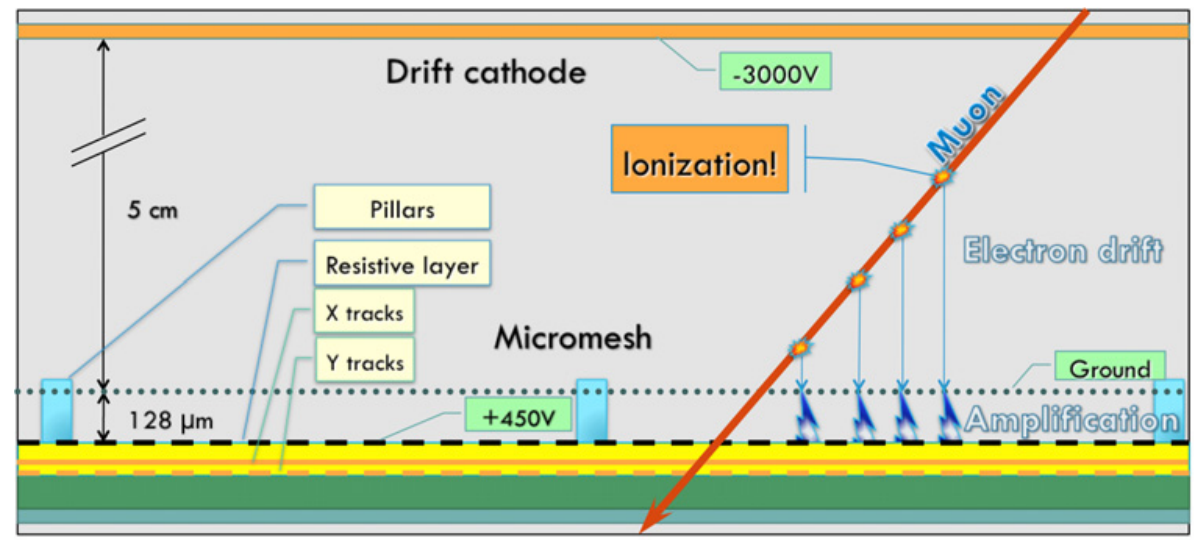

Figure 1. Schematic cross-section of the detector with its characteristic elements and functioning principle.

\section{MUST ${ }^{2}$ detector}

The Muon Survey Tomography based on Micromegas detectors for Unreachable Sites Technology $\left(\right.$ MUST $^{2}$ ) is a micro-pattern gaseous detector with two separated volumes: a conversion \& drift region, acting as Time Projection Chamber (TPC), and an amplification gap [5]. In the lower part of this gap lays the resistive layer, which serves as protection against sparks and reduces the output current that may damage the frontend electronics. Underneath the resistive layer, there are two superimposed layers with the readout tracks of $\mathrm{X}$ and $\mathrm{Y}$ axis respectively. The two planes are twisted $90^{\circ}$ with respect to each other to obtain a $2 \mathrm{D}$ measurement.

As seen in Fig. 1, when the ionizing particle enters in the conversion chamber, it ionizes the gas and the electrons generated will drift orthogonally towards the micromesh under the influence of the electric field in this region. Once the electron goes trough the micromesh, it enters in the amplification gap with a much stronger electric field, where undergoes acceleration that creates an avalanche effect that amplifies the charge. This charge, when collected by the resistive layer, will induce with its displacement a signal by capacitive coupling in both the micromesh and the readout tracks. The first one is used to command the acquisition and the second one for the image reconstruction.

By knowing the drift speed of the electron trough the gas and the arrival times of the ionizations, we are able to infer the zenithal angle of the trajectory.

Both the choice of gas and drift voltage for this topology are motivated by the need of a fast drift of the primary electrons in order to fit the whole signal in the $675 \mathrm{~ns}$ time window of the electronics, restriction imposed by the buffer size of the hybrid cards [6]. It is possible to determinate precisely the drift velocity of electrons in gas mixtures, for instance with the Magboltz code [7], to establish the operational parameter. The selected blend is composed of argon, CF4 and isobutene (88\%, 10\% and $2 \%$ respectively), which has a peak of drift speed at $600 \mathrm{~V} / \mathrm{cm}$ of more than $10 \mathrm{~cm} / \mu \mathrm{sec}$.

The gain of the detector is variable and is determined by the choice of the gas and the electric field in the amplification gap (controlled by the voltage of the resistive layer). The gain should be as high as possible but never exceed the Raether limit [8]. Otherwise the avalanche process becomes unstable and leads to the creation of a thin plasma filament that may damage irretrievably the resistive tracks. Typically the detector operates with a resistive voltage near the $420 \mathrm{~V}$ and it has a gain in the order of 10.000 [9]. 

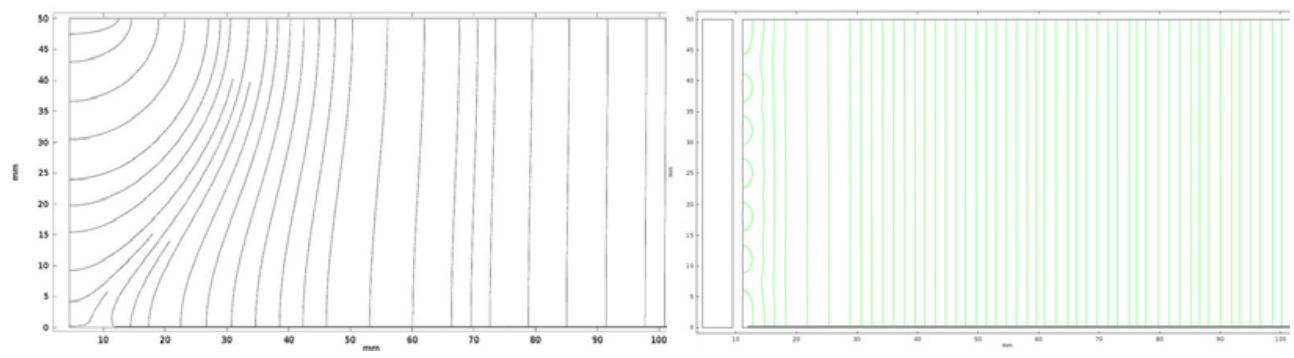

Figure 2. Field lines of the drift volume with (right) and without (left) the homogenizer device obtained with COMSOL Multiphysics ${ }^{\circledR}$.

The ratio between the two electric fields should be evaluated as well because it also influences the capability of the electrons to travel from the drift region to the amplification gap without being stopped (mesh electron transparency [10]), which creates a loss of information.

\section{Challenges}

The MUST $^{2}$ presents some distinguishing factors compared with other Micromegas detectors. It has a drift space of $5 \mathrm{~cm}$, which is about ten times the height of the most extended configuration for this kind of detector. On the one hand, this feature allows the reconstruction of the particle track with only one detection plane, with a resolution of 1 and 2 degrees in zenithal and azimuthal angles respectively. On the other hand, as shown in Fig. 2, the big distance from the cathode to the micromesh creates a non-homogeneous electric field all along the perimeter of the detector in the first $5 \mathrm{~cm}$ from the external frame.

To remove the possible artifacts induced by the local heterogeneities, an electric field homogenizer has been designed, based on COMSOL Multiphysics ${ }^{\circledR}$ simulations, and placed inside the TPC. This element has the double function to create a field gradation in the perimeter of the detector and, at the same time stimulates the gas distribution.

A new type of resistive layer has been recently developed by the Kobe University. The preliminary tests [11] with the latest Diamond-Like Carbon deposition resistive layers (DLC), have proven its robustness and good performance, creating a reliable alternative to the classical Carbon-Loaded Kapton layers (CLK). Some of their interesting features are the bigger charge collected by the hybrid cards, due to the bigger capacitance per unit of area of this layer, and the lower charge dispersion due to a smaller surface resistivity. They also show similar position resolution.

Electric field simulations of the amplification gap for both layers show that the DLC doesn't present the distortions in the amplification gap (see Fig. 3) due to the relief of the CLK.

A prototype of bulk-Micromegas with a DLC resistive foil has been already commissioned to the CERN PCB workshop and it's expected before the end of 2016.

\section{Self trigger}

Obtaining a trustworthy trigger signal to control the acquisition is one of the key settings of the detector. The device is able to acquire data using an external trigger, for instance created 

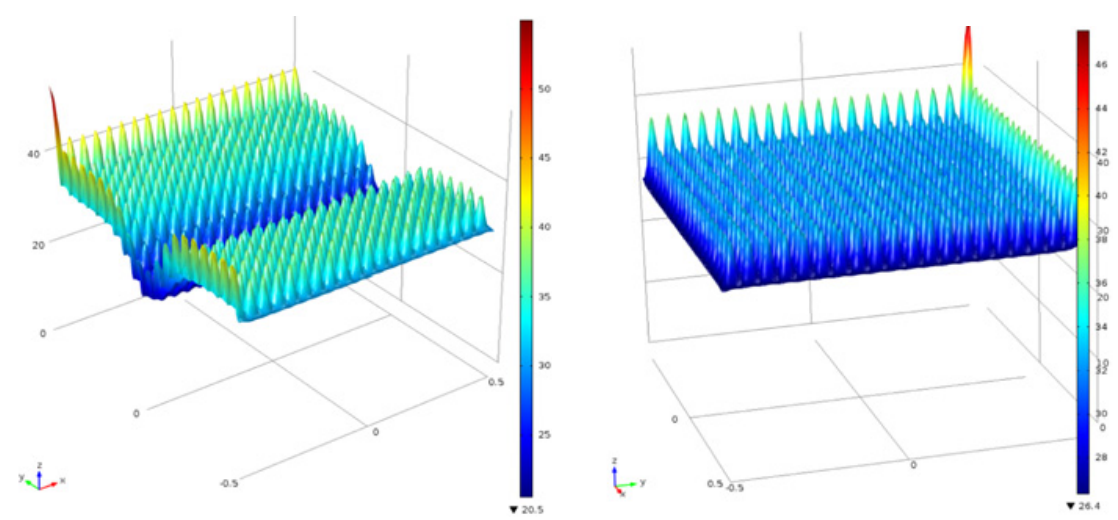

Figure 3. Simulated voltage in the micromesh plane with the CLK (left) and DLC (right) resistive layers obtained with COMSOL Multiphysics ${ }^{\circledR}$. X, Y, Z axis in $\mathrm{cm}$ and values in V.

with scintillators, but it has been conceived to be able to produce an internal trigger in order to operate in stand-alone mode. This reduces the number of peripherals and hence, the cost and volume of the detector.

The signal collected from the Micromesh is amplified and sent to a discriminator, where it is possible to set manually the trigger threshold. When the signal exceeds the fixed level, it creates a NIM pulse $[\mathrm{N}]$ that controls the FPGA and records the information of the readout tracks.

The Fig. 4 shows the preliminary results of the signal obtained from the mesh by measuring cosmic rays.

The MUST ${ }^{2}$ was placed between two plastic scintillators and the Fig. 4 shows the coincidence events between the three detectors in order to validate the signal obtained from the micromesh. The gas blend used in the experience is $\mathrm{Ar} / \mathrm{CO}_{2}$ (93:7), the drift electric field is set at $600 \mathrm{~V} / \mathrm{cm}$ and the voltage applied to the resistive layer ranges between 500 and $560 \mathrm{~V}$ (560V in the case of Fig. 4) to assess its effect in the gain. The micromesh signal is preamplified with an Ortec $142 \mathrm{C}$ and amplified with an Ortec 471.

While operating with only the resistive potential (which means no muons converted into electrons), a peak of noise appears at $\sim 204 \mathrm{mV}$. When the drift is activated, the events over $210 \mathrm{mV}$ start to appear and create for every voltage a similar shape, but with an amplitude proportional to the gain of the detector. This behavior is consistent with the functioning principle of the detector and the signal so generated may be used to trigger the acquisition with a delay smaller than the buffer time of the electronics.

Nevertheless, further analysis with different gases and experimental parameters needs to be done before we can fully validate the self-trigger feature.

\section{Summary and conclusion}

The current stage of the project consists of incorporating the improvements resulting of our experience with the physics simulation [12] and the first generation to overcome some limitations:

- A slight redesign of the mechanical components to minimize gas leaks.

- The replacement of the bulk Micromegas for a floating-mesh one. This will increase the ease of servicing in case of contamination of the detector, and will extend the lifetime of the detector. 


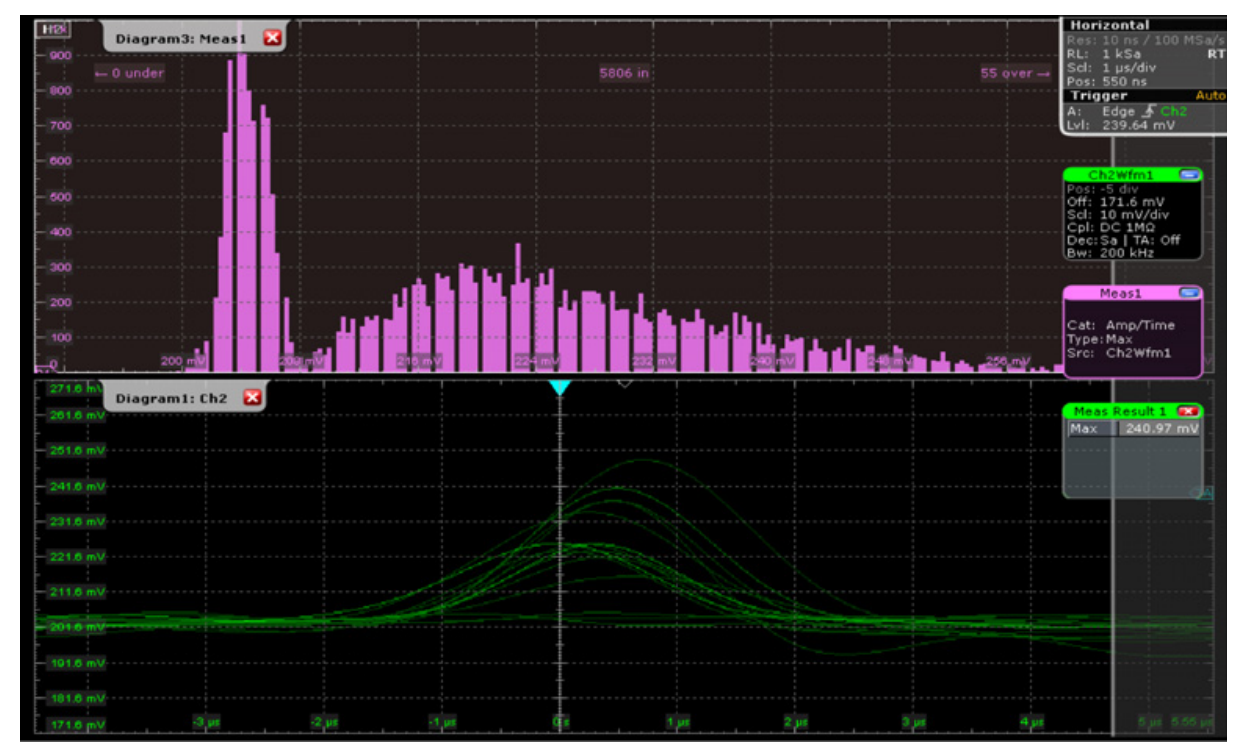

Figure 4. Oscilloscope's print-screen with the characteristic signal shape (bottom) and the histogram of signal amplitudes (top).

- The use of optic fiber equipment. It will notably increase the distance required between the detector and the data acquisition station. Hence reducing duplicity of materials in spread networks.

- The use of custom-made electronics that fits more precisely our needs.

Muon tomography, based on the absorption or attenuation of cosmic muons, is a mature technique still opened to innovation and the development of promising applications. The high temporal and spatial resolution of the new MUST ${ }^{2}$ camera allows to get more detailed images within less time. Thanks to its compact size and lightness, it is possible to deploy the device in places unreachable for other muon measurement equipment. It can be used in the framework of natural risk management and for Earth science and public works applications. This technology complements the existing ones (seismic, gravimetry, electric...). Once the camera prototype is fully operational, the collaboration will be focused on demonstrating this complementarity in real study cases.

The authors thank the priceless assistance of the CERN's PCB workshop and the RD51 collaboration for the development of micro-pattern gas detectors technologies for allowing us to work in their facilities with their equipment. Special thanks to R. de Oliveira, H. Muller and E. Oliveri for their commitment and dedication. This research was performed within the framework of a CIFRE grant (ANRT contract \#2015/0325) and supported by the European Fund for Regional Development (FEDER).

\section{References}

[1] E.P. George, Cosmic rays measure overburden of tunnel, Commonw. Eng. 455-457 (1955).

[2] L. Alvarez, et al., Search for hidden chambers in the pyramids, Science 167, 832-839 (1970). 
[3] Y. Giomataris, Ph. Rebourgeard, J.P. Robert, G. Charpak, A high-granularity positionsensitive gaseous detector for high particle-flux environments, Nucl. Instr. Meth. Phys. Res A 376, 29-35 (1996).

[4] I. Giomataris, R. De Oliveira, S. Andriamonje, S. Aune, G. Charpak, et al., Micromegas in a bulk, Nucl. Instr. Meth. A 560, 405-408 (2006).

[5] I. Lázaro et al., Muon telescope based on Micromegas detectors: from design to data acquisition, Proceedings of $i$-DUST conference (2014).

[6] J.-D. Berst et al., Recent developments and results on APV (DMILL) circuits for silicon and MSGC detectors, Proceedings of the Fifth Workshop on Electronics for LHC Experiments, CERN 99-09, CERN/LHCC/99-33, 108-112 (1999).

[7] S.F. Biagi, Monte Carlo simulation of electron drift and diffusion in counting gases under the influence of electric and magnetic fields, Nucl. Instr. and Meth. A 421, 234-240 (1999).

[8] T. Francke, Innovative Applications and Developments of Micro-Pattern Gaseous Detectors, ISBN 9781466660151 (2014).

[9] C. Adloff, M. Chefdeville, A. Espargiliere, R. Gaglione, Environmental study of a Micromegas detector, HAL Id: in2p3-00413881, pp.19 (2009).

[10] K. Nikolopoulos, P. Bhattachary, V. Chernyatin, R. Veenhof, Electron transparency of a Micromegas mesh. J. Instrum 6 (2011).

[11] P. Colas et al., Test of a new anode resistive coating for a Micromegas TPC, RD51 collaboration meeting (2015).

[12] U.S. NIM committee, Standard NIM Instrumentation System, DOE/ER-0457T (1990)

[13] F. Hivert et al., Muography sensitivity to hydrogeological rock density perturbation: roles of the absorption and scattering on the muon flux measurement reliability. Near Surf. Geophysics (accepted). 